\title{
Development of superparamagnetic iron oxide nanoparticles via direct conjugation with ginsenosides and its in-vitro study
}

Singh, Hina; Du, Juan; Singh, Priyanka; Mavlonov, Gafurjon Tom; Yi, Tae Hoo

Published in:

Journal of Photochemistry and Photobiology, B: Biology

Link to article, DOI:

10.1016/j.jphotobiol.2018.05.030

Publication date:

2018

Document Version

Peer reviewed version

Link back to DTU Orbit

Citation (APA):

Singh, H., Du, J., Singh, P., Mavlonov, G. T., \& Yi, T. H. (2018). Development of superparamagnetic iron oxide nanoparticles via direct conjugation with ginsenosides and its in-vitro study. Journal of Photochemistry and Photobiology, B: Biology, 185, 100-110. https://doi.org/10.1016/j.jphotobiol.2018.05.030

\section{General rights}

Copyright and moral rights for the publications made accessible in the public portal are retained by the authors and/or other copyright owners and it is a condition of accessing publications that users recognise and abide by the legal requirements associated with these rights.

- Users may download and print one copy of any publication from the public portal for the purpose of private study or research.

- You may not further distribute the material or use it for any profit-making activity or commercial gain

- You may freely distribute the URL identifying the publication in the public portal 


\section{Accepted Manuscript}

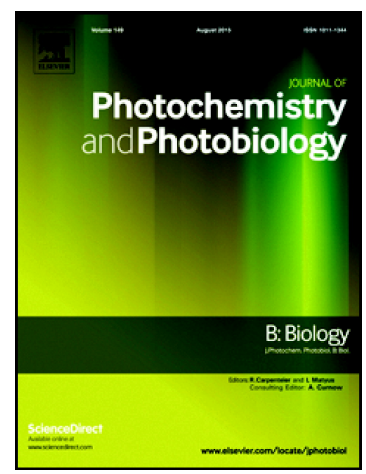

Hina Singh, Juan Du, Priyanka Singh, Gafurjon Tom Mavlonov, Tae Hoo Yi

Development of superparamagnetic iron oxide nanoparticles via direct conjugation with ginsenosides and its in-vitro study

PII: $\quad$ S1011-1344(18)30264-1

DOI: $\quad$ doi:10.1016/j.jphotobiol.2018.05.030

Reference: $\quad$ JPB 11264

To appear in: Journal of Photochemistry \& Photobiology, B: Biology

Received date: $\quad 7$ March 2018

Revised date: $\quad 3$ May 2018

Accepted date: $\quad 29$ May 2018

Please cite this article as: Hina Singh, Juan Du, Priyanka Singh, Gafurjon Tom Mavlonov, Tae Hoo Yi , Development of superparamagnetic iron oxide nanoparticles via direct conjugation with ginsenosides and its in-vitro study. Jpb (2017), doi:10.1016/ j.jphotobiol.2018.05.030

This is a PDF file of an unedited manuscript that has been accepted for publication. As a service to our customers we are providing this early version of the manuscript. The manuscript will undergo copyediting, typesetting, and review of the resulting proof before it is published in its final form. Please note that during the production process errors may be discovered which could affect the content, and all legal disclaimers that apply to the journal pertain. 
Development of superparamagnetic iron oxide nanoparticles via direct conjugation with ginsenosides and its in-vitro study

Hina Singh ${ }^{\mathrm{a}, \mathrm{b}}$, Juan Du ${ }^{\mathrm{a}, \mathrm{c}}$, Priyanka Singh ${ }^{\mathrm{a}, \mathrm{d}}$, Gafurjon Tom Mavlonov ${ }^{\mathrm{a}}$, Tae Hoo Yia ${ }^{\mathrm{a},}{ }^{*}$ drhoo@khu.ac.kr

${ }^{a}$ Department of Oriental Medicine Biotechnology, College of Life science, Kyung Hee University Global Campus, 1732 Deokyoungdaero, Giheung-gu, Yongin-si, Gyeonggi-do, 446-701, Republic of Korea

${ }^{\mathrm{b}}$ Division of Biomedical Sciences, School of Medicine, University of California, Riverside, USA, 92521

${ }^{\mathrm{c}}$ College of Food and Bioengineering, Zhengzhou University of Light Industry, Henan Province Collaborative Innovation Center for Food Production and Safety, Zhengzhou, 450001, China

${ }^{\mathrm{d} D T U}$ Biosustain, Novo Nordisk Foundation Center for Biosustainability, Technical University of Denmark, Lyngby 2800

${ }^{*}$ Corresponding author. 


\begin{abstract}
The current study focused on direct conjugation of superparamagnetic iron oxide nanoparticles (SPIONs) with ginsenosides $\mathrm{CK}$ and $\mathrm{Rg} 3$. The direct conjugation approach was low-cost, eco-friendly, simple, fast and high yield. The synthesized conjugates (SPION-CK and SPION-Rg3) were characterized by field emission transmission electron microscopy, dynamic light scattering, zeta potential, X-ray diffractometer, and magnetometer. The characterization results confirmed the formation of SPIONs conjugates. The maximum attaching percentage for ginsenosides to SPIONs was found to be $5 \%$. In vitro cytotoxicity assay in HaCaT keratinocyte cells revealed that the conjugates were non-cytotoxic to normal cells. Moreover, the anti-inflammatory activity of SPION-CK and SPION-Rg3 were investigated. The expression of reactive oxygen species (ROS) in lipopolysaccharide-activated RAW 264.7 (murine macrophage cells) were inhibited by SPIONs conjugates in a dose-dependent manner. In addition, SPION-CK and SPION-Rg3 significantly reduced the production of nitric oxide and inducible nitric oxide synthase (iNOS) in a dosedependent manner in the lipopolysaccharide-induced RAW 264.7 cells. Overall the results suggested that the SPIONs were conjugated with ginsenosides $\mathrm{CK}$ and $\mathrm{Rg} 3$ by using direct conjugation approach were non-cytotoxic and can be used as a carrier for intracellular release of ginsenosides in inflammatory diseases.
\end{abstract}

Keywords: Superparamagnetic iron oxide nanoparticles, direct conjugation, ginsenoside CK, ginsenoside Rg3 


\section{Introduction}

Superparamagnetic iron oxide nanoparticles (SPIONs) were highly researched in biomedical and nanomedicine field. The inherent biodegradable and biocompatible properties of SPIONs make them highly advantageous in nanomedicine. Additionally, the magnetic properties of SPIONs make them promising candidates to be used for biomedical applications such as drug delivery, diagnosis, and therapy. [1]

There is a plethora of synthetic methods for SPIONs like physical, chemical and biological have been reported in the literature. [2,3] Further, SPIONs with bare surface tend to agglomerate due to strong magnetic attraction among particles. [4] SPIONs can exhibit low probability of reaching the desired target, non-specific interaction with proteins that can cause aggregation, adsorption of opsonins leading to phagocytosis and subsequent clearance from the body, and toxicity issues. [5] So, these can be avoided by conjugation or surface functionalization of SPIONs. Moreover, conjugation will provide stability, protection against clearance, increases bioavailability, exhibit low toxicity, and offer the potential for further conjugation. [6]

Several strategies have been applied for conjugation. The most common approach is the linker chemistry, where chemical linkers cross-link nanoparticles and conjugating molecules. [7] Even though several chemical linkers are available, the chemical linker approach suffers from many disadvantages. For e.g. special reaction conditions, low conjugation efficiency, multiple cleaning steps and use of toxic chemical like 1-ethyl-3-(dimethylaminopropyl) carbodiimide (EDC) and N-hydroxysuccinimide (NHS). [8] On the other hand specific molecular recognition based on biotin-streptavidin is another common method. [9] The shortcomings of this approach are the need of complex procedure, expensive reagents, low specificity, low yield etc. Some other conjugation strategy already reported are often complex and require strict control of conditions. [10]

Developing conjugation strategies for high-quality nanoparticles remains a challenge and essential need with regards to their applicability in the biomedical field. Despite several conjugation strategies have been employed its needs to develop simple and efficient conjugation method. Considering all the limitations of existing methods, in this study direct conjugation approach with natural medicinal compounds has been developed which is low cost, ecofriendly, simple, fast and high yield. In the present study, the synthesized SPIONs were stabilized with natural medicinal compounds using direct conjugation approach thereby avoiding the need for expensive stabilizing agents which makes the process often complex. [10] Direct conjugation method, is conjugation method were the surface of nanoparticles can be directly coated with any compounds of interest. However, there have been no investigations on 
the direct conjugation with natural medicinal compounds like ginsenosides despite some obvious advantages. In case of SPIONs the direct conjugation strategies have only been explored for polymers or surfactant like poly ethyl glycol, oleic acid etc. $[10,11]$ The direct conjugation process also eliminates the use of chemical linkers, simplifies the conjugation process and increases the efficiency of the conjugation.

Panax ginseng is traditionally used as a medicinal herb in Korea, China, and Japan. [12] Ginsenosides are the main active components in $P$. ginseng. Minor ginsenosides like $\mathrm{CK}$ and $\mathrm{Rg} 3$ have several pharmacological activities like anti-inflammation, anticancer, antitumor, cardioprotective, neuroprotective properties etc. $[13,14,15,16,17]$ Although the improved efficacy of ginsenosides have been reported, their target-based delivery, as well as their poor bioavailability and absorption, remain major drawbacks for use in clinical trials. $[18,19]$ By reviewing these reports, ginsenosides have been selected as a candidate for natural medicinal compound-based nanoparticles study. And it was considered that, if successful, this approach can be further applied to any natural sugar-based nanoparticles.

In this study, the objective was to develop direct conjugation method of SPIONs with ginsenosides. Further, the biological activities of synthesized SPIONs conjugates were explored to see if SPIONs may serve as an effective carrier for ginsenosides. Biological activities thereby synthesized SPIONs conjugates were tested by antiinflammatory activities.

\section{Materials and methods}

\subsection{Materials}

Iron (III) chloride hexahydrate $\left(\mathrm{FeCl}_{3} 6 \mathrm{H}_{2} \mathrm{O}, 97 \%\right)$, Iron (II) chloride tetrahydrate $\left(\mathrm{FeCl}_{3} 4 \mathrm{H}_{2} \mathrm{O}, 99 \%\right)$ and Dimethyl sulfoxide (DMSO, 99\%) were purchased from Samchun Chemicals (Seoul, Korea). Sodium hydroxide (NaOH, 93-100\%) was purchased from Duksan Pure Chemicals (Gyeonggi-do, Korea). Ginsenosides samples (CK and Rg3) (purity >90\%, TLC) was kindly supported by Professor Feng-Xie Jin (Dalian Polytechnic University, China). All the other chemicals which have been used were purchased from Sigma-Aldrich (St. Louis, MO, USA).

$\mathrm{HaCaT}$ cells, originated from human epidermal keratinocytes, were purchased from Sciencell (Carlsbad, CA, USA). HCT-116 cells, derived from human colon cancer cells and RAW 264.7 cells, derived from murine macrophage cells were obtained from the Korean Cell Line Bank (KCLB, Seoul, South Korea). Dulbecco's modified eagle medium (DMEM), Roswell Park Memorial Institute medium (RPMI-1640), fetal bovine serum (FBS), penicillin and 
streptomycin were purchased from Gibco BRL (Grand Island, NY, USA). Unless otherwise mentioned, solvents were purchased from Samchun Chemicals (Seoul, Korea).

\subsection{Synthesis of SPIONs}

A brief overview of the synthesis method of SPIONs was illustrated in Fig. 1. The synthesis of SPIONs was achieved via co-precipitation method. The SPIONs synthesis was based on sodium hydroxide $(\mathrm{NaOH})$ mediated precipitation from 2:1 molar mixture of $\mathrm{Fe}^{3+}$ and $\mathrm{Fe}^{2+}$ salts (both in chloride form). Briefly, $5.4 \mathrm{~g}$ of $\mathrm{FeCl}_{3}$ hexahydrate (or $3.2 \mathrm{~g}$ of anhydrous) and $1.9 \mathrm{~g}$ of $\mathrm{FeCl}_{2}$ tetrahydrate (or $1.8 \mathrm{~g}$ of anhydrous) dissolved in $1 \mathrm{~L}$ of distilled water. After that, the mixture was kept under vigorously mixing and $40 \mathrm{~mL}$ of $2 \mathrm{M} \mathrm{NaOH}$ was added slowly. The pH of reaction mixture was adjusted at 12 with $\mathrm{NaOH}$. After 5 min agitation, the reaction mixture transferred to a water bath set at $65^{\circ} \mathrm{C}$ for $30 \mathrm{~min}$. SPIONs pellet collected by means of neodymium magnet N52 (5x5x2.5 cm; 5000 gausses) and washed two times with distilled water. The SPIONs were suspended in $200 \mathrm{~mL}$ of water and kept in the refrigerator $\left(4^{\circ} \mathrm{C}\right)$ before using.

\subsection{Synthesis of SPION-Ginsenosides (SPION-GS) conjugates}

For the synthesis of SPIONs conjugates with ginsenosides (SPION-GS), $250 \mathrm{mg}$ of ginsenosides (CK and Rg3) dissolved in $50 \mathrm{~mL}$ of $30 \%$ ethanol. Then, $2 \mathrm{~g}$ of SPIONs suspended in $200 \mathrm{~mL}$ of distilled water was washed two times and sonicated for 10 min by means UP100H ultrasonic device (Hielscher, Germany) (Fig. 2). Ginsenosides solution and SPIONs mixed and additionally sonicated for $10 \mathrm{~min}$. Then the mixture is vigorously shaken at room temperature at least for $4 \mathrm{~h}$. Resulted SPION-GS washed with distilled water three times. The nanoparticle was collected by means of the external magnet. SPION-GS were freeze-dried and kept at $4{ }^{\circ} \mathrm{C}$. This freeze-dried powder was used for further experiments.

\subsection{Determine the content of ginsenosides in the SPION-GS}

To determinate the percentage of ginsenosides attached to SPIONs, $10 \mathrm{mg}$ of SPION-GS (freeze-dried powder) was suspended in $0.5 \mathrm{~mL}$ of ethanol (95\%):1\% acetic acid solution. Mixture sonicated for 3 min until dissolved completely. Finally, the eluate and nanoparticles pellet separated by centrifugation at $12000 \mathrm{rpm}$ for $5 \mathrm{~min}$. Quantitation of ginsenosides in eluates carried out by using thin layer chromatography (TLC) using TLC Kiesel gel $60 G \mathrm{~F}_{254}$ plates $(10 \times 10 \mathrm{~cm}$, Merck, Germany). Separately, an equal volume of eluates and standard (ginsenosides 
CK and Rg3) were spotted on to the TLC plates. The plates were developed using chloroform/methanol/water (65/35/10). Finally, the chromatogram visualized using $10 \%$ sulfuric acid followed by drying using the dryer, until the spots appear. Photocopy of chromatogram processed by means of precision scanning software Quantity One ${ }^{\circledR}$ (Bio-Rad, USA). For calculation of the percentage of attached natural medicinal compounds following formula has been used:

Attaching percentage $=$ Spot intensity of sample $\times$ Concentration of standard $\times$ Volume of sample

Spot intensity of sample $\times$ mass of sample

\subsection{Characterization of SPIONs and SPION-GS}

Formation of colloidal SPIONs and SPIONs conjugates were determined through UV-vis spectrophotometer. Metal nanoparticles strongly interact with specific wavelengths of light and show different peaks. The optical absorption spectra of SPIONs and SPIONs conjugated samples were recorded by using UV-vis (Optizen POP; Mecasys; Daejeon, Korea). The spectrum was recorded between the wavelength range 200-400 nm. The morphology, elemental distribution and selected area diffraction (SAED) pattern of SPIONs and SPION-GS were analyzed through field emission transmission electron microscopy (FE-TEM), with a JEM-2100F (JEOL, Tokyo, Japan) instrument operated at $200 \mathrm{kV}$. The samples were prepared by placing a drop of samples solution (in water) on a carbon-coated copper grid and subsequently drying in an oven at $60{ }^{\circ} \mathrm{C}$ before transferring it to the microscope. The hydrodynamic size, polydispersity index, and zeta potential were measured in triplicate using Zetasizer Nano ZS90 (Malvern Instruments, UK) at $25{ }^{\circ} \mathrm{C}$ and at a 12 angle. As a reference dispersive medium pure water with refractive index 1.3328 , viscosity 0.8878 , and dielectric constant 78.3 was used. For hydrodynamic size and zeta potential analysis, the samples were suspended in water and then used. Synthesized SPIONs and SPIONs were also examined by scanning electron microscope (SEM, LEO SUORA 55, GENESIS 2000, Carl Zeiss, Jena, Germany).

The crystalline structure was determined by X-ray diffraction (XRD), which shows a different peak at different intervals. The XRD analyses were performed on X-ray diffractometer, D8 Advance, (Bruker), Germany, operated at $40 \mathrm{kV}, 40 \mathrm{~mA}$, with $\mathrm{CuK} \alpha$ radiation, at a scanning rate of $6^{\circ} / \mathrm{min}$, step size 0.02 , over the $2 \theta$ range of $20-80^{\circ}$. The magnetic properties were studied using a magnetometer equipment (vibrating sample magnetometer (VSM), Lake Shore Cryotronics, USA) based on the flux meter method connected to a data acquisition computing system (PC), a wide range of temperature $(5-300 \mathrm{~K})$ and at fields ranging from 0 to $10 \mathrm{k}$ Oe. For SEM, XRD and magnetometer 
analysis, powder (freeze-dried) form of samples has been used. The superparamagnetic activity of synthesized SPIONs was analyzed by placing the SPIONs suspension in presence of an external magnetic field.

\subsection{Cytotoxicity evaluation}

To test the in vitro cytotoxicity MTT assay was carried out against HaCaT and HCT-116 cells. HaCaT cells were cultured in DMEM, and HCT-116 cells were cultured in RPMI-1640 both medium supplemented with 10\% heatinactivated FBS and $1 \%$ penicillin and streptomycin $(\mathrm{P} / \mathrm{S})$ at $37{ }^{\circ} \mathrm{C}$ in an atmosphere containing $5 \% \mathrm{CO}_{2}$. Both cells were seeded at a density of $1 \times 10^{5}$, cells per well in a 96-well microtiter plate (Corning Costar, Lowell, NY) and grown for $24 \mathrm{~h}$. When cells reached over $80 \%$ confluence, the treatment was performed with various concentrations of SPIONs, CK, Rg3, SPIONs conjugated with ginsenoside CK (SPION-CK) and SPIONs conjugated with ginsenoside RG3 (SPION-Rg3). The concentration in all experiments was based on the percentage of ginsenosides attached to the SPIONs. After $24 \mathrm{~h}$ of treatment, MTT at a final concentration of $0.1 \mathrm{mg} / \mathrm{mL}$ was added to each well and further incubated for $3 \mathrm{~h}$; then, the formazan was dissolved in DMSO and the absorbance of each well was determined on microplate reader (Molecular Devices Filter Max F5; Sunnyvale, CA, USA) at a wavelength of $520 \mathrm{~nm}$.

\subsection{Antioxidant activity}

\subsubsection{Diphenyl-1-picrylhydrazyl scavenging activity}

Free radical scavenging activity was determined by Diphenyl-1-picrylhydrazyl (DPPH) assay. A $20 \mu \mathrm{l}$ of different concentration of samples were placed in a 96-well plate and $180 \mathrm{~mL}$ of 2, 2-diphenyl-1-picrylhydrazyl $(0.2$ $\mathrm{mM}$ ) in methanol was also added. The plate was standing at $37^{\circ} \mathrm{C}$ for $30 \mathrm{~min}$ in dark, and the absorbance was measured by a microplate reader (Molecular Devices Filter Max F5; Sunnyvale, CA, USA) at the wavelength of $520 \mathrm{~nm}$. Each sample was separately tested at a various concentration from $1 \mu \mathrm{g} / \mathrm{mL}$ to $250 \mu \mathrm{g} / \mathrm{mL}$. Arbutin was used as positive control.

\subsubsection{Measurement of intracellular reactive oxygen species (ROS)}

To determine the level of ROS, RAW 264.7 (murine macrophage) cells were plated in 96-well plate at a density $1 \times 10^{5}$ cells/well, supplemented with DMEM, $10 \%$ (V/V) FBS and $1 \%(\mathrm{~V} / \mathrm{V}) \mathrm{P} / \mathrm{S}$ and incubated for $24 \mathrm{~h}$ at $37^{\circ} \mathrm{C}$ in a humidified incubator $\left(5 \% \mathrm{CO}_{2}\right.$ and $95 \%$ air). After $24 \mathrm{~h}$, the cells were stimulated with $1 \mu \mathrm{g} / \mathrm{mL}$ of 
lipopolysaccharide (LPS, Sigma Aldrich Co., USA) in presence and absence of SPIONs, CK, Rg3, and SPION-GS conjugated samples (SPION-CK and SPION-Rg3) and incubated for $24 \mathrm{~h}$ at $37^{\circ} \mathrm{C}$. After $24 \mathrm{~h}$ of treatment, the cells were stained with $30 \mu \mathrm{M} 2^{\prime}, 7^{\prime}$-dichlorofluorescein diacetate (DCF-DA) for $30 \mathrm{~min}$ at $37^{\circ} \mathrm{C}$. Then the cells were rinsed twice with PBS and subjected to a microplate reader (Molecular Devices Filter Max F5; Sunnyvale, CA, USA).

\subsection{Anti-inflammatory activity on RAW 264.7 cells}

\subsubsection{Cell viability and measurement of nitrite level}

To determine the cell viability and level of nitrite in cultured cells, the RAW 264.7 were seeded at density of 1 x 105 cells/well in 96-microplate for $24 \mathrm{~h}$ and pretreated with $1 \mu \mathrm{g} / \mathrm{mL}$ lipopolysaccharide LPS and with various concentration (1, 10 and $100 \mu \mathrm{g} / \mathrm{mL})$ of SPIONs, CK, Rg3, SPION-CK and SPION-Rg3 for $24 \mathrm{~h}$. Cells without LPS stimulation was used as normal. After $24 \mathrm{~h}$, a total $100 \mu \mathrm{L}$ of culture supernatant was transferred to new microplate and then mixed with equal volume (100 $\mu \mathrm{L})$ of Griess reagent (Sigma Aldrich Co., USA), and the absorbance was measured at $540 \mathrm{~nm}$ against a standard sodium nitrite curve using a microplate reader (Molecular Devices Filter Max F5; Sunnyvale, CA, USA). The left $100 \mu \mathrm{L}$ of culture supernatant was used to perform MTT assay.

\subsubsection{Reverse transcription-polymerase chain reaction (RT-PCR)}

Based on the NO and ROS results SPIONs has not been selected for further study. RAW 264.7 cells were stimulated with LPS $(1 \mu \mathrm{g} / \mathrm{mL})$ in the presence or absence of CK, Rg3, SPION-CK, and SPION-Rg3. Total RNA was isolated by harvesting the whole-cell lysate using TRIZOL reagents (Invitrogen Life Technologies, Carlsbad, CA, USA) according to the manufacturer's instructions. The RNA ( $2 \mu \mathrm{g})$ was reverse transcribed using 200 units of reverse transcriptase and $0.5 \mu \mathrm{g} / \mu \mathrm{L}$ oligo-(dT) $)_{15}$ primer. This reaction was performed at $42{ }^{\circ} \mathrm{C}$ for 60 min and was terminated at $94^{\circ} \mathrm{C}$ for $5 \mathrm{~min}$, for 30 times. PCR amplification of cDNA template was performed using PCR premix and following primers: glyceraldehyde-3-phosphate dehydrogenase (GAPDH) and inducible nitric oxide synthase (iNOS). The primer sequences are listed in Table 1. For GAPDH, the reaction was performed at $42{ }^{\circ} \mathrm{C}$ for 60 min and was terminated a $94{ }^{\circ} \mathrm{C}$ for $5 \mathrm{~min}$, for 30 times. For iNOS, the reaction was performed at $94{ }^{\circ} \mathrm{C}$ for $5 \mathrm{~min}$ for one cycle and then $94{ }^{\circ} \mathrm{C}$ for $1 \mathrm{~min}, 56^{\circ} \mathrm{C}$ for $30 \mathrm{~s}$, and $72{ }^{\circ} \mathrm{C}$ for $1 \mathrm{~min}$ for 30 cycles. The amplified polymerase chain reaction (PCR) products were separated by $2.0 \%$ agarose gel electrophoresis with ethidium bromide (Sigma-Aldrich) staining, at $100 \mathrm{~V}$ for 20 min. GAPDH was used as an internal control. 


\subsection{Statistical analysis}

All the results were representative of three independent experiments and were expressed as mean $\pm \mathrm{SD}$. The results were determined using Graph Pad Prism 5 (GraphPad Software, Inc., CA, USA). Comparison of control and treatment groups was performed using ANOVA variance analysis, and the statistical significance was analyzed by Duncan's test. $P<0.05$ was significant.

\section{Results}

\subsection{Characterization of SPIONs and SPION-GS}

The reduction of iron precursors to nanoparticles were seen by UV-vis spectroscopy. UV-vis spectra of SPIONs and SPIONs conjugated samples were shown in Fig. 3. The absorbance peak of SPIONs appears at $210 \mathrm{~nm}$ which is typical for iron oxide nanoparticles. The UV-vis analysis peak for SPIONs conjugated samples showed the highest peak at $375 \mathrm{~nm}$. The peak shifted towards red because the surface of the SPIONs in SPION-GS has been coated with ginsenosides. The red shift in UV-vis peaks further confirms the successful conjugation. The morphology of SPIONs conjugates was determined by FE-TEM instrument. The FE-TEM images of the SPIONs, SPION-CK, and SPIONRg3 proclaimed a roughly spherical morphology (Fig. 4a-c). In these micrographs, the apparent flocculation of particles is an artifact of drying during TEM grid sample preparation. The elemental mapping data clearly show iron oxide nanoparticles distributed evenly in the SPION-GS (Fig. 4d-f). The SAED pattern of SPIONs and SPION-GS was shown in Fig. S1a-c. The ring pattern in SAED confirms the crystalline nature of SPIONs and SPION-GS.

The average size $( \pm$ SD) of synthesized SPIONs, SPION-CK and SPION-Rg3 were $42.9 \pm 0.3 \mathrm{~nm}, 61.2 \pm 1.6$ $\mathrm{nm}$ and $68.3 \pm 1.7 \mathrm{~nm}$. The zeta potential value $( \pm \mathrm{SD})$ of SPIONs, SPION-CK and SPION-Rg3 were determined to be $-26.2 \mathrm{mV} \pm 5.9,+37.5 \pm 6.8 \mathrm{mV}$ and $+27.9 \pm 5.8 \mathrm{mV}$ and $+38.7 \pm 4.1 \mathrm{mV}$. The zeta potential value of core SPIONs was above $-25 \mathrm{mV}$, indicating the stability of SPIONs for a long time in solution. Moreover, the electrostatic repulsive force among the negatively charged surface of the SPIONs affords high stability to the colloidal solution by preventing them from agglomerating in the colloid state. On the other hand, positive zeta potential value was obtained for SPIONs conjugated samples. This is a result of the coating of the surface of SPIONs with natural medicinal compounds. The zeta potential value of SPIONs conjugated samples was above $+25 \mathrm{mV}$, indicating the stability of samples for a long time in solution. The zeta potential value lays within the stable range, signifying that the SPIONs and SPIONs conjugation formation lead to the formation of the stable system. The hydrodynamic size and zeta potential value of 
SPIONs and conjugates were shown in Fig. S1d-i. The average size and zeta potential values obtained for SPIONs and SPIONs conjugated samples is indicated in Table 2. The surface morphology of the samples was also examined by SEM is shown in the Fig. S2a-c. The EDAX spectrum of samples obtained using SEM showed Peaks around 0.9 and $6.1 \mathrm{keV}$ which are related to the iron oxide nanoparticles elements (Fig. S2d-f).

XRD pattern of SPIONs showed the peaks at $31.2^{\circ}, 36.81^{\circ}, 44.7^{\circ}, 59.3^{\circ}$ and $65.1^{\circ}$ corresponding to the (220), (311), (400), (511) and (440) reflections facets of the cubic crystalline structure (Fig. 5a). This pattern was similar to the Braggs's reflection of pure crystalline magnetite. All these distinct diffraction peaks correspond to the reflection of face-centered cubic (fcc) crystalline magnetite $\left(\mathrm{Fe}_{3} \mathrm{O}_{4}\right)$ standard (JCPDS, PDF\#26-1136), indicated the formation of SPIONs. The SPION-GS exhibited intense peaks in the whole spectrum of $2 \theta$ value ranging from $20^{\circ}$ to $80^{\circ}$ corresponding to (220), (311), (400), (511) and (440) reflections (Fig 5b). This pattern was similar to the Braggs's reflection of pure magnetite $\left(\mathrm{Fe}_{3} \mathrm{O}_{4}\right)$ indicating that the purified product is composed of pure crystalline magnetite. The diffraction peaks were consistent with the standard data files (PDF\#26-1136). The absence of any other peaks in XRD pattern indicated the high purity of SPIONs and SPION-GS.

The magnetic hysteresis (MH) curve for core SPIONs and SPION-GS were presented in (Fig. 5c,d). The VSM determined Ms value for SPIONs was $70.2 \mathrm{emu} / \mathrm{g}$ which is about $31.1 \%$ of the standard value $220 \mathrm{emu} / \mathrm{g}$ for pure iron phase. The Ms value was more than $52 \mathrm{emu} / \mathrm{g}$, indicating the good magnetic saturation of synthesized SPIONs. [20,21] The Ms value for SPIONs conjugated samples was $38.1 \mathrm{emu} / \mathrm{g}$ which is about $17.3 \%$ of the standard value of pure iron phase. The Ms value obtained for the SPIONs conjugated samples was less than SPIONs, indicating that the surface of SPIONs has been coated with the natural medicinal compound. Similar MH curve was obtained for SPIONs conjugated with ginsenoside CK, ginsenoside Rg3 and quercetin. Additionally, SPIONs shows superparamagnetic properties. The suspension of SPIONs in water becomes strongly magnetized in the presence of an external magnetic field. As soon they come to the contact of an external magnetic field they behave like a permanent magnet. This test confirms that the synthesized SPIONs were superparamagnetic in nature (Fig S3).

\subsection{Determination and validation of ginsenosides in SPION-GS}

Fig. 6, Showed the TLC result of SPION-GS. The content of ginsenosides attached is around $\sim 5 \%$ (4.9\% for SPION-Rg3 and 5.3\% for SPION-CK). The appearance of similar TLC spots and same retention factor (Rf) peaks compared to that of control (CK and Rg3) confirms that conjugation has occurred and ginsenosides did not chang after 
conjugation. SPIONs was good dispersible in water, but after conjugation with ginsenosides show very low solubility in water. This is because the surface of SPIONs gets coated with ginsenosides and the surface become hydrophobic. Adding non-ionic detergent Tween 20 (polysorbate 20, pharmacopeia approved emulsifier; Sigma-Aldrich) up to $0.1 \%$ gives good solubility of SPION-GS.

\subsection{In vitro cytotoxicity}

To observe the cytotoxicity of samples, the cell viability in HaCaT keratinocyte cells and HCT-116 colon cancer cells was examined by MTT assay. Fig. 7 indicates, SPIONs at the concentration $(1-100 \mu \mathrm{g} / \mathrm{mL})$ had no effect on the viability of HaCaT keratinocyte cells and HCT-116 colon cancer cells. But, when the concentration of CK range from 10-100 $\mu \mathrm{g} / \mathrm{mL}$, the cell viability on HaCaT cells was slightly affected whereas SPION-CK did not affect the cell viability at the concentration up to $100 \mu \mathrm{g} / \mathrm{mL}$. In case of HCT-116 cells, although CK showed a huge cytotoxic effect from the concentration $50 \mu \mathrm{g} / \mathrm{mL}$, SPION-CK showed little cytotoxicity at the same concentrations (Fig. 7a,b). On HaCaT cells, Rg3 showed cytotoxicity at the concentrations range from 1-100 $\mu \mathrm{g} / \mathrm{mL}$ whereas SPION-Rg3 did not affect the cell viability. But on HCT-116 cells, Rg3 and SPION-Rg3 showed similar cytotoxicity in the concentrationdependent manner (Fig. 7c,d). A sigmodal curve for MTT assay is represented in Fig. S4.

\subsection{Antioxidant activity}

\subsubsection{Diphenyl-1-picrylhydrazyl scavenging activities}

The free radical scavenging activity of samples was determined by DPPH assay. Only SPIONs have no free radical scavenging activity. As shown in Fig. S5a, ginsenoside CK have low scavenging activity but SPION-CK has considerably high free radical scavenging activity. The DPPH inhibition activity of SPION-CK was measured at 250 $\mu \mathrm{g} / \mathrm{mL}$ by $72 \%$ which is significantly higher than that of CK. The scavenging activity of $\operatorname{Rg} 3$ is very low which is slightly enhanced in case of SPION-Rg3. The scavenging activity of SPION-Rg3 was measured at $250 \mu \mathrm{g} / \mathrm{mL}$ by $33.5 \%$, which is higher than that of Rg3 (Fig. S5b). Overall there is no DPPH scavenging activity on SPIONs treatment. However, SPION-GS have the free radical scavenging activity. The $\mathrm{IC}_{50}$ values of all the samples is presented in Table 3.

\subsubsection{ROS production}


To investigate the effects of SPIONs, CK, Rg3, SPION-CK, and SPION-Rg3 on LPS-induced ROS generation, the cellular level of ROS secretion was measured. Fig. 8 indicates that SPIONs seems to have no effect on ROS production. When cells treated with CK and SPION-CK showed significant inhibition of ROS at $100 \mu \mathrm{g} / \mathrm{mL}$ which were $5.5 \%$ and $17.7 \%$. On the other hand, $\operatorname{Rg} 3$ seems to have no effect on attenuation of ROS production. However, increase in ROS levels was quenched by SPION-Rg3 treatment at $10 \mu \mathrm{g} / \mathrm{mL}$ and $100 \mu \mathrm{g} / \mathrm{mL}$ by $80.6 \%$ and $16.0 \%$.

\subsection{Anti-inflammatory activity on RAW 264.7 cells}

\subsubsection{Cell viability and NO production}

To check the anti-inflammatory effect, cell viability and NO assay was performed on LPS-induced RAW 264.7 cells. LPS treatment can induce NO production in RAW 264.7 cells. Fig. 9 indicates that cell viability and LPS-induce NO production were not affected by SPIONs treatment. Treatment with CK and SPION-CK caused significant decrease cell viability at the concentration $100 \mu \mathrm{g} / \mathrm{mL}$. Although cytotoxicity was observed at the concentration 100 $\mu \mathrm{g} / \mathrm{mL}$, the NO production was significantly inhibited by CK and SPION-CK at the concentration $10 \mu \mathrm{g} / \mathrm{mL}$ (Fig. 9a). $\mathrm{Rg} 3$ and SPION-Rg3 did not affect the cell viability at concentrations ranging from 1-100 $\mu \mathrm{g} / \mathrm{mL}$. However, NO production was significantly decreased by Rg3 and SPION-Rg3 in a concentration-dependent manner (Fig. 9c).

\subsection{2. mRNA level of iNOS measured by RT-PCR}

After stimulation with LPS $(1 \mu \mathrm{g} / \mathrm{mL})$, the iNOS mRNA expression was measured RAW 264.7 cells which were treated with CK, SPION-CK, Rg3, and SPION-Rg3. As shown in Fig. 10, iNOS mRNA expression was upregulated in LPS induced RAW 264.7 cells, while treatment with samples inhibited the overproduction of iNOS mRNA expression. The elevated iNOS mRNA expression was decreased in a concentration-dependent manner. In Rg3 and SPION-Rg3 treated cells the iNOS mRNA level was inhibited by $43.7 \%$ and $44.4 \%$ at $100 \mu \mathrm{g} / \mathrm{mL}$. In case of CK and SPION-CK treated cells, iNOS mRNA level was investigated at the concentration of $1 \mu \mathrm{g} / \mathrm{mL}$ and $10 \mu \mathrm{g} / \mathrm{mL}$. Due to the cytotoxic effect of CK and SPION-CK at the concentration $100 \mu \mathrm{g} / \mathrm{mL}$, this concentration was eliminated for RTPCR analysis. mRNA level of iNOS was inhibited by treatment with CK and SPION-CK by $47.9 \%$ and $45.8 \%$ at 10 $\mu \mathrm{g} / \mathrm{mL}$. LPS, as well as tested samples, did not affect mRNA expression of the housekeeping gene GAPDH.

\section{Discussion}


The aim of this study was to develop a low cost, eco-friendly, simple, fast and high yield direct conjugation method of superparamagnetic iron oxide nanoparticles (SPIONs) with natural medicinal compounds. Primarily, using direct conjugation approach SPIONs conjugation with ginsenosides has been done. In previous reports, toxic and expensive chemicals such as EDC/NHC or glutaraldehyde have been used for conjugation, which often raises the environmental issue. [22] In this study, no expensive or toxic chemicals were used for conjugation, indicated that the direct conjugation method was cheap and eco-friendly. Additionally, fewer steps make the direct conjugation method simple. The previous study on conjugation of Rg3 with SPIONs suggested that the conjugation method takes 12-15 h and needs special instruments for purification steps. [5] In the present investigation, the direct conjugation method took 1-4 h making the process fast. Further, no special instruments were used for purification steps. Previously reported, conjugation methods required complex conditions like high temperature, column for purification, dark and unoxygenated environment. $[23,24]$ On the other hand, the direct conjugation could be performed at an ambient condition like room temperature and in presence of oxygen.

Despite the numerous reported conjugation methods in the literature, there is an ever-increasing demand for the development of simple and efficient conjugation strategy. [6] Present results suggested that a simple, cheap, fast and eco-friendly direct conjugation approach has been developed. In addition, the direct conjugation method showed high reproducibility. The direct conjugation approach was used to achieve ginsenosides (CK and $\mathrm{Rg} 3$ ) conjugation to SPIONs. TLC results demonstrated that the binding of natural medicinal compounds i.e. ginsenosides Rg3 and CK have occurred and the structure of the compound did not change after conjugation (Fig. 6). Notably, the binding percentage of ginsenosides to SPIONs was found to be 5\%. Present results were similar to the previously reported work which showed the attaching percentage for ginsenosides to SPIONs was 5\%. [14] Their data also suggested that SPIONs conjugated with ginsenoside $\mathrm{Rg} 3$ was soluble in acid. In this study, the results showed that SPIONs were good dispersible in water, but after conjugation with ginsenosides CK and $\operatorname{Rg} 3$ the solubility in water was decreased. Adding non-ionic detergent Tween 20 (polysorbate 20, pharmacopeia approved emulsifier) up to $0.1 \%$ give good solubility of SPIONs conjugated samples (SPION-CK and SPION-Rg3).

Further, the characterization results indicated the appropriate characteristics of SPIONs and SPIONs conjugated samples. Previously, several unique shapes of SPIONs were discovered for e.g. nanorod, nanocubes, nano-husk, spherical, distorted cubes, flower-shaped etc. [25] In the present study, TEM images confirmed the morphology of synthesized SPIONs and SPIONs conjugated samples were roughly spherical in shape. The size and stabilizing factors 
of SPIONs and SPIONs conjugated samples was observed in DLS and zeta potential. In this work, the average size ( \pm SD) of synthesized SPIONs was $42.9 \pm 0.3 \mathrm{~nm}$. But after conjugation with ginsenosides, the average size was increased (Table 4). The increase in size resulted because the surface of SPIONs has been coated with ginsenosides. This characterization proved that the conjugation was successful. [22,26] As reported by Cheng et al., 2015 [27] the stability of SPIONs deteriorated when the SPIONs was conjugated with curcumin. The zeta potential value of curcumin conjugated SPIONs was decreased to $-0.01 \pm 0.01 \mathrm{mV}$ suggested the highly unstable nature of conjugates. The present results supported the formation of colloidal stable SPIONs conjugates. The zeta potential value of SPION-CK and SPION-Rg3 were $+37.8 \pm 6.8 \mathrm{mV}$ and $+27.4 \pm 5.8 \mathrm{mV}$, respectively. This zeta potential value obtained for SPIONGS lays at the margin of stable range, signifying that the SPIONs conjugates formation leads to the formation of relatively stable system. [28] The magnetic saturation value for SPIONs was determined to be $70.2 \mathrm{emu} / \mathrm{g}$, which is considered as good magnetic property. [10] On the other hand, in SPIO-GS the Ms value were decreased to 38.1 emu/g. The decrease in Ms value can be attributed to the fact that SPIONs surface has been coated with ginsenosides. Similar results were reported by Sun et al., 2008 [29] their results suggested that the Ms value of SPIONs was decreased by $50 \%$ after the coating with different surfactants like sodium oleate, polyvinyl alcohol, and starch. This result confirmed the conjugation was successful. The XRD results further provided the evidence that the synthesized SPIONs and SPION-GS were crystalline structure. Based on results, an effective direct conjugation method has been developed. This conjugation strategy can be applied for any natural medicinal compounds or drugs containing hydroxyl (-OH), amine (-NH2), sulfhydryl (-SH), phosphate (PO43-), carboxylic (-COOH) and vicinal hydroxyl groups or their combination.

After the successful synthesis of SPION-CK and SPION-Rg3, they have been explored for biomedical applications. SPIONs are considered as biocompatible because they can be cleared from the body by opsonization or through degradation via the body's metabolism forming iron ions that are then included in normal body metabolism e.g. used by erythrocytes to form hemoglobin [30]. SPIONs are one of the only US food and drug administration (FDA) approved nanoparticles for their use as MRI contrast agents of the liver and as iron supplements [31,33]. In the present study in-vitro cytotoxic activity shows that SPIONs were non-cytotoxic in nature (Fig 7). On the other hand, cytotoxicity was observed in case of ginsenosides CK and $\mathrm{Rg} 3$ but these ginsenosides have already been reported for their anticancer and antitumor activities [13,14]. Ginsenoside CK also seems to damage $\mathrm{HaCaT}$ cells at $50 \mu \mathrm{g} / \mathrm{mL} \&$ $100 \mu \mathrm{g} / \mathrm{mL}$ concertation. 
The DPPH scavenging results showed that the SPIONs conjugated samples have antioxidant activity (Fig. S5). The free radicals scavenging might be due to the electron transfer from the nanoparticles system to the free radical located at the nitrogen atom in DPPH [33]. Recently some reports showed the anti-inflammatory effect of nanoparticles. [34] In this study, it has been also evaluated the anti-inflammatory effect of SPIONs conjugated samples on LPS-induced RAW-264.7 cells. As expected, intracellular reactive oxygen species (ROS) and nitric oxide (NO) is stimulated by LPS treatment. ROS and NO production in LPS-induced RAW 264.7 cells were markedly decreased by SPIONs conjugated samples (Fig. 8 \& Fig. 19. Further, RT-PCR analysis revealed that all CK, SPION-CK, Rg3, and SPION-Rg3 significantly decreased LPS-induced mRNA expression levels of iNOS (Fig. 10). Based on this result, the SPIONs conjugated samples may be a potential therapeutic for the treatment of inflammatory diseases. However, further in vivo study is required to determine how these SPIONs conjugated samples inhibit the overexpression of inflammatory mediators.

Direct conjugation method is fast and effective, however further work is needed to determine other factors that influence the activity of SPIONs conjugates like the release of natural medicinal compounds depending on $\mathrm{pH}$, temperature and applied a magnetic field. Also, there is need investigate issues regarding in vivo toxicity, systemic degradation and bioclearence of SPIONs.

\section{Conclusion}

The present study demonstrated the development of direct conjugation method of SPIONs with natural medicinal compounds like ginsenoside CK and ginsenoside Rg3. SPIONs were conjugated with CK and $\operatorname{Rg} 3$ in by using direct conjugation method, which is low cost, eco-friendly, simple, fast and high yield. The characterization results showed the small size, improved stability and superparamagnetic activity of synthesized SPIONs conjugates. The SPION-GS showed anti-oxidant properties. Anti-inflammatory activity was confirmed by ROS, NO, iNOS tests. Present results supported the potential of SPIONs to act as an effective carrier for natural medicinal compounds. Direct conjugation approach for SPIONs has been developed that could also be used for other natural medicinal compounds to improve their bioavailability, targeted delivery, minimizing dose concentration and unwanted toxicity to normal cells. However, further study is needed, regarding in vivo toxicity and bioclearence of SPIONs.

\section{Acknowledgments}


This work was conducted under the industrial infrastructure program (No. N0000888) for fundamental technologies which is funded by the Ministry of Trade, Industry, and Energy (MOTIE, Korea). 


\section{References}

1. Sun, C.; Lee, J. S.; Zhang, M. Magnetic nanoparticles in MR imaging and drug delivery. Adv. Drug Deliv. Rev., 2008, 60, 1252-1265.

2. Cuenya, B.R. Synthesis and catalytic properties of metal nanoparticles: Size, shape, support, composition, and oxidation state effects. Thin Solid Films, 2010, 518, 3127-3150.

3. Narayanan, K. B.; Sakthivel, N. Biological synthesis of metal nanoparticles by microbes. Adv. Colloid Inter. Sci., 2010, 156, 1-13.

4. Xia, T.; Wang, J.; Wu, C.; Meng, F.; Shi, Z.; Lian, J.; Meng, J. Novel complex-coprecipitation route to form high quality triethanolamine-coated $\mathrm{Fe}_{3} \mathrm{O}_{4}$ nanocrystals: their high saturation magnetizations and excellent water treatment properties. Cryst. Eng. Comm., 2012, 14, 5741-5744.

5. Emerich, D. F.; Thanos, C. G. The pinpoint promise of nanoparticle-based drug delivery and molecular diagnosis. Biomol. Eng., 2006, 23, 171-184

6. Lu, A. H.; Salabas, E. E.; Schüth, F. Magnetic nanoparticles: synthesis, protection, functionalization, and application. Angew. Chem. Int. Ed., 2007, 46, 1222-1244.

7. Wilchek, M.; Bayer, E. A.; Livnah, O. Essentials of biorecognition: The (strept) avidin-biotin system as a model for protein-protein and protein-ligand interaction. Immunol. Lett., 2006, 103, 27-32.

8. Xu, Y.; Baiu, D. C.; Sherwood, J. A.; McElreath, M. R.; Qin, Y.; Lackey, K. H.; Bao, Y. Linker-free conjugation and specific cell targeting of antibody functionalized iron-oxide nanoparticles. J. Mater. Chem. B., 2014, 2, 6198-6206.

9. Xu, Y.; Qin, Y.; Palchoudhury, S.; Bao, Y. Water-soluble iron oxide nanoparticles with high stability and selective surface functionality. Langmuir, 2011, 27, 8990-8997.

10. Mascolo, M. C.; Pei, Y.; Ring, T. A. Room temperature co-precipitation synthesis of magnetite nanoparticles in a large pH window with different bases. Materials, 2013, 6, 5549-5567.

11. Barreto, A. C.; Santiago, V. R.; Freire, R. M.; Mazzetto, S. E.; Denardin, J. C.; Mele, G.; Carbone, L. Magnetic nanosystem for cancer therapy using oncocalyxone A, an antitumor secondary metabolite isolated from a Brazilian plant. Int. J. Mol. Sci., 2013, 14, 18269-18283. 
12. Singh, P.; Singh, H.; Castro-Aceituno, V.; Ahn, S.; Kim, Y. J.; Yang, D. C. Bovine serum albumin as a nanocarrier for the efficient delivery of ginsenoside compound $\mathrm{K}$ : preparation, physicochemical characterizations and in vitro biological studies. RSC Adv., 2017, 7, 15397-15407.

13. Ahn, S.; Siddiqi, M. H.; Noh, H. Y.; Kim, Y. J.; Kim, Y. J.; Jin, C. G.; Yang, D. C. Anti-inflammatory activity of ginsenosides in LPS-stimulated RAW 264.7 cells. Sci. Bull., 2015, 60, 773-784.

14. Park, S. M.; Choi, M. S.; Sohn, N. W.; Shin, J. W. Ginsenoside Rg3 attenuates microglia activation following systemic lipopolysaccharide treatment in mice. Biol. Pharma. Bull., 2012, 35, 1546-1552.

15. Shergis, J. L.; Di, Y. M.; Zhang, A. L.; Vlahos, R.; Helliwell, R.; Ye, J. M.; Xue, C. C. Therapeutic potential of Panax ginseng and ginsenosides in the treatment of chronic obstructive pulmonary disease. Complement. Ther. Med., 2014, 22, 944-953.

16. Zhang, Z.; Du, G. J.; Wang, C. Z.; Li, Z.; Yuan, C. S. Ginsenoside compound K inhibits colorectal tumorigenesis via multiple pathways including ATM/p53-p21. Cancer Res., 2012, 72, 1685-1685.

17. Kim, J. Cardioprotective Effect of the Mixture of Ginsenoside Rg-3 and CK on Contractile Dysfunction of Ischemic Heart. Ginseng Res., 2007, 31, 23-33.

18. Kim, H. S.; Lee, E. H.; Ko, S. R.; Choi, K. J.; Park, J. H.; Im, D. S. Effects of ginsenosides Rg 3 and Rh 2 on the proliferation of prostate cancer cells. Arch. Pharm. Res., 2004. 27, 429-435.

19. Davis, J. M.; Murphy, E. A.; Carmichael, M. D. Effects of the dietary flavonoid quercetin upon performance and health. Curr. Sports Med. Rep., 2009, 8, 206-213.

20. Thorek, D. L.; Chen, A. K.; Czupryna, J.; Tsourkas, A. Superparamagnetic iron oxide nanoparticle probes for molecular imaging. Ann. Biomed. Engg., 2006, 34, 23-38.

21. Kumar, S.; Layek, S.; Pandey, B.; Verma, H. C. Magnetic structure of Fe-Fe oxide nanoparticles made by electrodeposition. Int. J. Eng. Sci. Technol, 2009, 2,66-72.

22. Tseng, S. H.; Chou, M. Y.; Chu, I. M. Cetuximab-conjugated iron oxide nanoparticles for cancer imaging and therapy. Int. J. Nanomedicine, 2012, 10, 3663-3685.

23. Yang, R.; Chen, D.; Li, M.; Miao, F.; Liu, P.; Tang, Q. 20 (s)-ginsenoside Rg3-loaded magnetic human serum albumin nanospheres applied to HeLa cervical cancer cells in vitro. Bio-med. Mater. Eng., 2014, 24, 19911998. 
24. Yu, M. K.; Park, J.; Jon, S. Targeting strategies for multifunctional nanoparticles in cancer imaging and therapy. Theranostics. 2012, 2, 3-44.

25. Sayed, F. N.; Polshettiwar, V. Facile and sustainable synthesis of shaped iron oxide nanoparticles: effect of iron precursor salts on the shapes of iron oxides. Sci. Rep. 2015, 5, 9733.

26. Yang, J.; Luo, Y.; Xu, Y.; Li, J.; Zhang, Z.; Wang, H.; Zhang, G. Conjugation of iron oxide nanoparticles with RGD-modified dendrimers for targeted tumor MR imaging. ACS Appl. Mater. interfaces, 2015, 7, $5420-5428$.

27. Cheng, K. K.; Chan, P. S.; Fan, S.; Kwan, S. M.; Yeung, K. L.; Wáng, Y. X. J.; Baum, L. Curcuminconjugated magnetic nanoparticles for detecting amyloid plaques in Alzheimer's disease mice using magnetic resonance imaging (MRI). Biomaterials, 2015, 44, 155-172.

28. Hanaor, D.; Michelazzi, M.; Leonelli, C.; Sorrell, C. C. The effects of carboxylic acids on the aqueous dispersion and electrophoretic deposition of ZrO2. J. Eur. Ceram. Soc., 2012, 32, 235-244.

29. Sun, C.; Lee, J. S.; Zhang, M. Magnetic nanoparticles in MR imaging and drug delivery. Adv. Drug Del. Rev., 2008, 60, 1252-1265.

30. Owens, III D.E.; Peppas, N.A. Opsonization, biodistribution, and pharmacokinetics of polymeric nanoparticles. International journal of pharmaceutics. 2006 Jan 3;307(1):93-102.

31. Reimer, P.; Balzer T.; Ferucarbotran (Resovist): a new clinically approved RES-specific contrast agent for contrast-enhanced MRI of the liver: properties, clinical development, and applications. European radiology. 2003 Jun 1;13(6):1266-76.

32. Spinowitz, B.S.; Kausz, A.T.; Baptista, J.; Noble; S. D.; Sothinathan, R.; Bernardo, M. V.; Brenner, L.; Pereira, B. J. Ferumoxytol for treating iron deficiency anemia in CKD. Journal of the American Society of Nephrology. 2008 Aug 1;19(8):1599-605.

33. Bhattacharya, K.; Gogoi, B; Buragohain A.K.; Deb, P.; 2014. $\mathrm{Fe}_{2} \mathrm{O}_{3} / \mathrm{C}$ nanocomposites having distinctive antioxidant activity and hemolysis prevention efficiency. Mater. Sci. Eng. C. 2014, 42, 595-600.

34. Singh, P.; Ahn, S.; Kang, J. P.; Veronika, S.; Huo, Y.; Singh, H.; Yang, D. C. In vitro anti-inflammatory activity of spherical silver nanoparticles and monodisperse hexagonal gold nanoparticles by fruit extract of Prunus serrulata: a green synthetic approach. Artif. Cells Nanomed. Biotechnol, 2017, 1-11. 
Graphical abstract: Conjugation scheme of ginsenosides with superparamagnetic iron oxide nanoparticles (SPIONs).

Fig.1. Schematic procedure of synthesis of superparamagnetic iron oxide nanoparticles (SPIONs).

Fig. 2. Stepwise process of direct conjugation of SPIONs with ginsenosides.

Fig. 3. Ultraviolet-visible spectra of SPIONs (a) and SPION-GS (b). For SPION-CK and SPION-Rg3 similar XRD and $\mathrm{MH}$ cure obtained, hence only one result has been shown.

Fig. 4.TEM images of SPIONs (a), SPION-CK, (b) and SPION-Rg3 (c). Elemental mapping results indicate the distribution of elements in respective nanoproducts, SPIONs (d), SPION-CK (e), SPION-RG3 (f).

Fig. 5. X-Ray diffraction pattern and Magnetic hysteresis curve of SPIONs (a \& c) and SPION-GS (b \& d). For SPION-CK and SPION-Rg3 similar XRD and MH cure obtained, hence only one result has been shown.

Fig. 6. TLC based determination of ginsenosides content in SPIONs conjugated with ginsenosides.

TLC image (a), peak obtained in quantity by One-Bio-Rad software for CK (b), CK eluate (c), $\operatorname{Rg} 3$ (d), and $\operatorname{Rg} 3$ eluate (e). The TLC image was analyzed using quantity One-Bio-Rad software. Each experiment was performed independently in triplicate and data expressed as mean $\pm \mathrm{SD}$.

Fig. 7. In vitro effect of SPIONs, CK, SPION-CK, Rg3, and SPION-Rg3 on cell viability of HaCaT keratinocyte cells (a \& c) and HCT-116 colon cancer cells (b \& d).

For cell cytotoxicity assay, $\mathrm{HaCaT}$ and HCT-116 cells were incubated for $24 \mathrm{~h}$ with various concentration $(1,10,50$, $100 \mu \mathrm{g} / \mathrm{mL}$ ) of superparamagnetic iron oxide nanoparticles (SPIONs), ginsenoside CK and SPIONs conjugated with ginsenoside CK (SPION-CK). Cell viability was determined by MTT assay. Data shown represent the mean values of three experiments $\pm \mathrm{SD}$

Fig. 8. Effect of SPIONs, CK, SPION-CK, Rg3, and SPION-Rg3 on the ROS production in RAW 264.7 cells. 
For ROS determination, cells were treated with various concentration $(1,10,100 \mu \mathrm{g} / \mathrm{mL})$ of SPIONs, ginsenoside CK, SPION-CK, Rg3, SPION-Rg3 and stimulated with LPS (1 $\mu \mathrm{g} / \mathrm{mL})$ for $24 \mathrm{~h}$. The ROS were detected by DCF-DA assay as describes in methods. Data shown represent the mean values of three experiments \pm SD. ${ }^{\# \#} P<0.001$ when compared with LPS untreated group (normal). ${ }^{*} P<0.05, * * * P<0.001$ as compared to the group treated with LPS alone.

Fig. 9. Effect of SPIONs, CK, SPION-CK, Rg3, and SPION-Rg3 on cell viability (a \& c) and nitric oxide production (b \& d) in RAW 264.7 cells.

For cell viability and nitric oxide assay, RAW 264.7 cells treated with various concentration $(1,10,100 \mu \mathrm{g} / \mathrm{mL}) \mathrm{of}$ SPIONs, ginsenoside CK, SPION-CK, ginsenoside Rg3, SPION-Rg3 and stimulated with LPS (1 $\mu \mathrm{g} / \mathrm{mL})$ for $24 \mathrm{~h}$. The concentration of nitrite was determined as described in methods. Data shown represent the mean values of three experiments $\pm \mathrm{SD} .{ }^{\# \# \#} P<0.001$ when compared with LPS untreated group (normal). $* * P<0.01, * * * P<0.001$ as compared to the group treated with LPS alone.

Fig. 10. Effect of ginsenoside CK, SPION-CK, ginsenoside Rg3, SPION-Rg3, on the expression of iNOS mRNA level.

RAW 264.7 cell were treated with different concentration $(1,10 \mu \mathrm{g} / \mathrm{mL}$ for $\mathrm{CK}, \mathrm{SPION}-\mathrm{CK}$ and $10,100 \mu \mathrm{g} / \mathrm{mL}$ for Rg3, SPION-Rg3) and stimulated with LPS (1 $\mu \mathrm{g} / \mathrm{mL})$ for $24 \mathrm{~h}$. The mRNA expression level was determined by RTPCR analysis and compared with those of GAPDH. ${ }^{\# \#} P<0.001$ when compared with LPS untreated group (normal). $* P<0.05$ and $* * * P<0.001$ as compared to the group treated with LPS alone. 
Table 1. PCR primer sequence

\begin{tabular}{|c|c|c|}
\hline \multirow{2}{*}{ Primer name } & Orientation & Primer sequence 5' to 3' orientation \\
\hline \multirow{2}{*}{ GAPDH } & Forward & TGCACCACCAACTGCTTAGC \\
\cline { 2 - 3 } & Reverse & GGCATGGACTGTGGTCATGAG \\
\hline \multirow{2}{*}{ iNOS } & Forward & ACCCAAGGTCTACGTTCAGG \\
\cline { 2 - 3 } & Reverse & CGCACATCTCCGCAAATGTA \\
\hline
\end{tabular}


Table 2. Particles size distribution and zeta potential measurement of SPIONs and SPION-GS.

\begin{tabular}{|c|c|c|}
\hline Samples & Average size $( \pm \mathrm{SD})$ & Zeta Potential $( \pm \mathrm{SD})$ \\
\hline SPIONs & $42.9 \pm 0.3 \mathrm{~nm}$ & $-26.2 \pm 5.9 \mathrm{mV}$ \\
\hline SPION-CK & $61.2 \pm 1.6 \mathrm{~nm}$ & $+37.5 \pm 6.8 \mathrm{mV}$ \\
\hline SPION-Rg3 & $68.3 \pm 1.7 \mathrm{~nm}$ & $+27.9 \pm 5.8 \mathrm{mV}$ \\
\hline
\end{tabular}

The particles size distribution was investigated by dynamic light scattering (DLS) analysis, and the surface charge and stability of nanoparticles was investigated by zeta potential, DDW was used as a dispersive medium. Each experiment was performed in triplicate. The data were represented as mean \pm SD. SPIONs; superparamagnetic iron oxide nanoparticles, SPION-CK; SPIONs conjugated with ginsenoside CK, SPION-Rg3; SPIONs conjugated with ginsenoside $\operatorname{Rg} 3$ 
Table 3. Antioxidant activity (\% of DPPH discoloration) of SPIONs, CK, Rg3 and SPIONS-GS.

\begin{tabular}{|c|c|c|}
\hline Samples & Concentration $(\mu \mathrm{g} / \mathrm{mL})$ & DPPH radical inhibition (\%) \\
\hline \multirow[t]{5}{*}{ SPIONs } & 1 & $1.5 \pm 5.4$ \\
\hline & 10 & $1.6 \pm 5.1$ \\
\hline & 100 & $1.9 \pm 3.8$ \\
\hline & 200 & $5.3 \pm 2.4$ \\
\hline & 250 & $7.2 \pm 3.5$ \\
\hline \multirow[t]{5}{*}{$\mathrm{CK}$} & 1 & $2.3 \pm 3.2$ \\
\hline & 10 & $9.7 \pm 1.8$ \\
\hline & 100 & $16.6 \pm 2.8$ \\
\hline & 200 & $19 \pm 3.6$ \\
\hline & 250 & $21.1 \pm 4.2$ \\
\hline \multirow[t]{5}{*}{ SPION-CK } & 1 & $3.1 \pm 0.8$ \\
\hline & 10 & $8.2 \pm 0.6$ \\
\hline & 100 & $31.5 \pm 3.2$ \\
\hline & 200 & $47.9 \pm 6$ \\
\hline & 250 & $72 \pm 5.8$ \\
\hline \multirow[t]{5}{*}{$\operatorname{Rg} 3$} & 1 & $0.04 \pm 3.9$ \\
\hline & 10 & $0.72 \pm 1.2$ \\
\hline & 100 & $1.1 \pm 2.7$ \\
\hline & 200 & $4.1 \pm 2.1$ \\
\hline & 250 & $7.4 \pm 3$ \\
\hline \multirow[t]{5}{*}{ SPION-Rg3 } & 1 & $0.37 \pm 0.2$ \\
\hline & 10 & $0.92 \pm 0.5$ \\
\hline & 100 & $2.5 \pm 1.3$ \\
\hline & 200 & $10.7 \pm 0.7$ \\
\hline & 250 & $33.5 \pm 1.3$ \\
\hline
\end{tabular}

Antioxidant activity was determined by DPPH assay. Each experiment was performed independently in triplicate, and the data are indicated as mean \pm standard deviation. 
Table 4. Characterization of SPIONs and SPION-GS.

\begin{tabular}{|c|c|c|c|c|c|c|c|}
\hline Samples & Shape & $\begin{array}{l}\text { Average } \\
\text { size }\end{array}$ & $\begin{array}{c}\text { Zeta } \\
\text { Potential }\end{array}$ & Magnetization & $\begin{array}{c}\text { Attaching } \\
(\%)\end{array}$ & Solubility & $\begin{array}{l}\text { Colloidal } \\
\text { stability }\end{array}$ \\
\hline SPIONs & Spherical & $\begin{array}{c}42.9 \pm 0.3 \\
\mathrm{~nm}\end{array}$ & $\begin{array}{l}-26.2 \pm \\
5.9 \mathrm{mV}\end{array}$ & $70.2 \mathrm{emu} / \mathrm{g}$ & & in water & Stable \\
\hline $\begin{array}{l}\text { SPION- } \\
\text { CK }\end{array}$ & Spherical & $\begin{array}{c}61.2 \pm 1.6 \\
\mathrm{~nm}\end{array}$ & $\begin{array}{c}+37.8 \pm \\
6.8 \mathrm{mV}\end{array}$ & 3 & & $\begin{array}{c}\text { In } 0.1 \% \\
\text { Tween } 20\end{array}$ & Stable \\
\hline $\begin{array}{l}\text { SPION- } \\
\text { Rg3 }\end{array}$ & Spherical & $\begin{array}{c}68.3 \pm 1.7 \\
\mathrm{~nm}\end{array}$ & $\begin{array}{c}+27.4 \pm \\
5.8 \mathrm{mV}\end{array}$ & 38.1 en & $4.8 \pm 0.3$ & $\begin{array}{c}\text { In } 0.1 \% \\
\text { Tween } 20\end{array}$ & Stable \\
\hline
\end{tabular}

SPIONs, Superparamagnetic iron oxide nanoparticles; SPION-CK, SPIONs conjugated with ginsenoside CK;

SPION-Rg3, SPIONs conjugated with ginsenoside Rg3; NA, not applicable. 


\section{Highlights}

- Simple, cheap, ecofriendly and high yield direct conjugation method has been developed.

- Direct conjugation method was used for superparamagnetic iron oxide nanoparticles (SPIONs) conjugation.

- Direct conjugation method was successful in conjugating SPIONs with ginsenosides CK and Rg3.

- The ginsenosides conjugates were nontoxic to HaCaT keratinocyte cells.

- The conjugates showed anti-inflammatory effects in RAW 264.7 cells. 


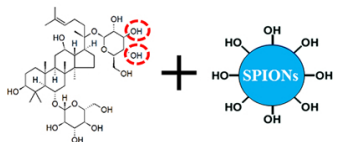

Ginsenosides

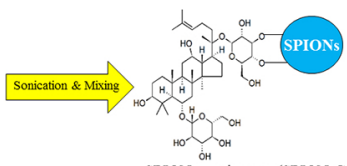

SPIONs conjugates (SPION-GS)

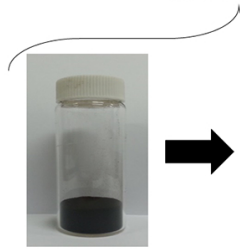

SPION-GS without magnet

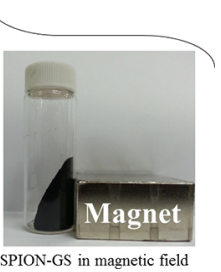

\section{Graphics Abstract}




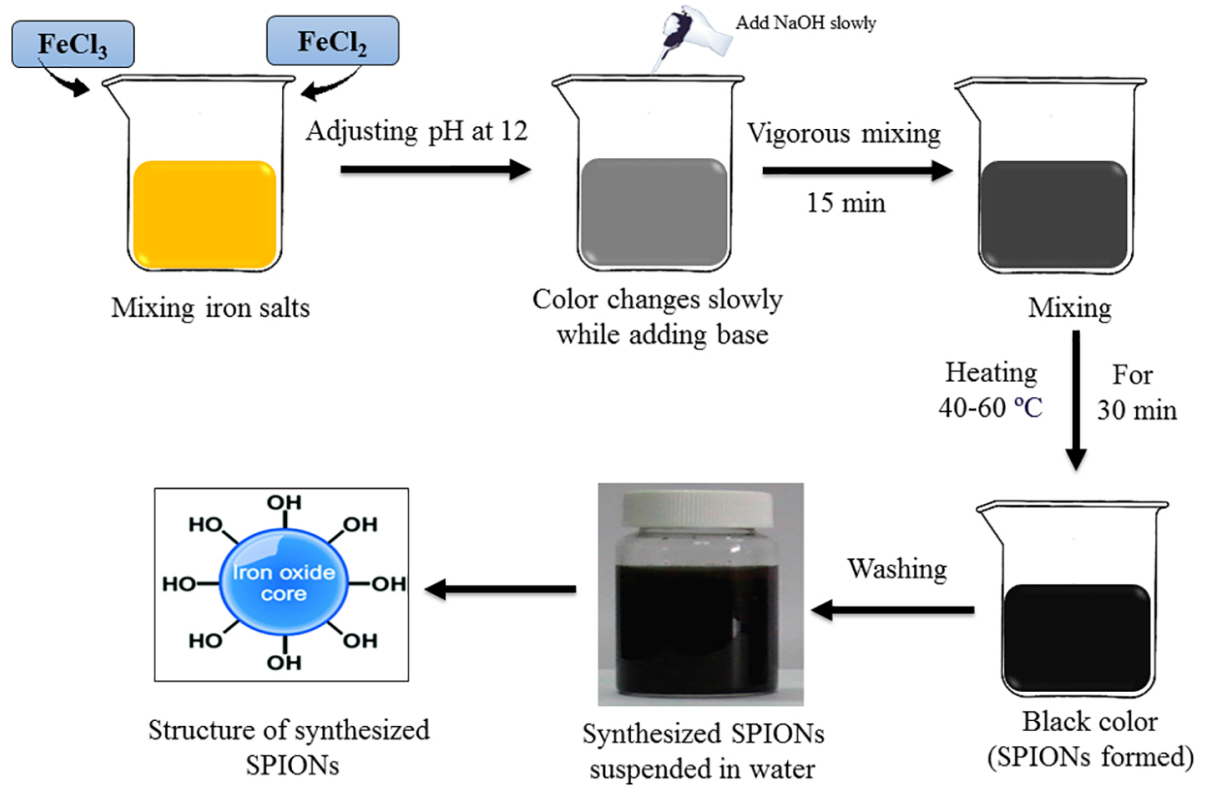

Figure 1 


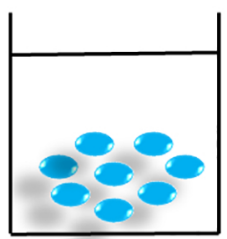

SPIONs in water
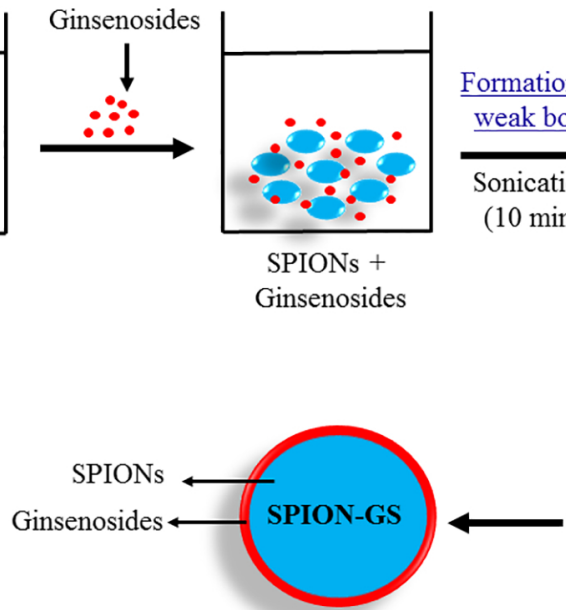

Structure of SPION-GS
Formation of weak bond Sonication (10 min) SPIONs + Ginsenosides

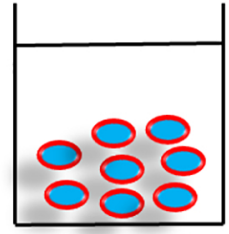

SPION-GS

Washing \& drying
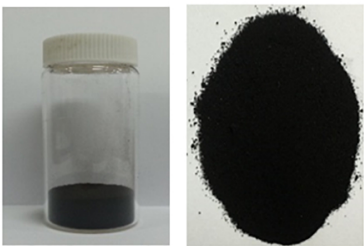

Freeze dry powder of conjugates

Figure 2 


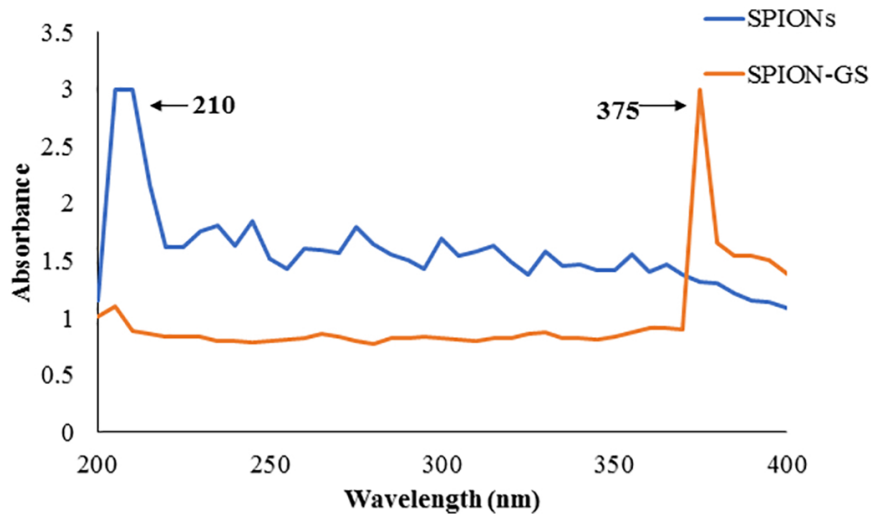

Figure 3 
(a)

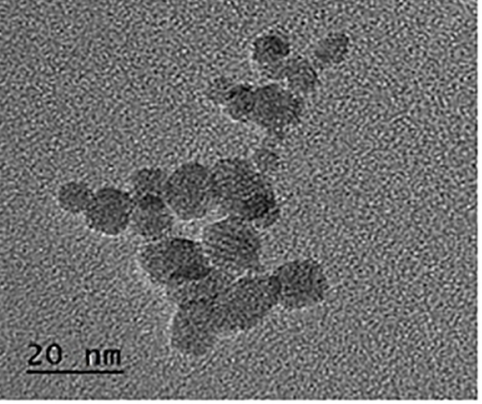

(b)

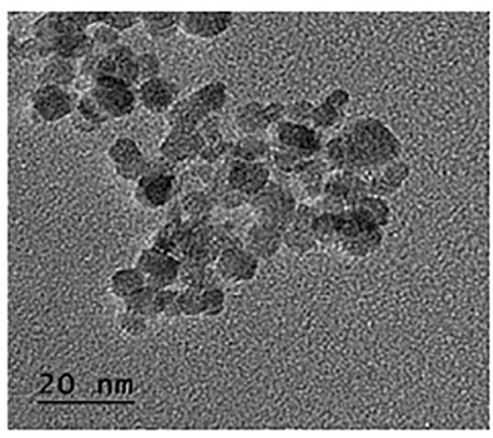

(c)

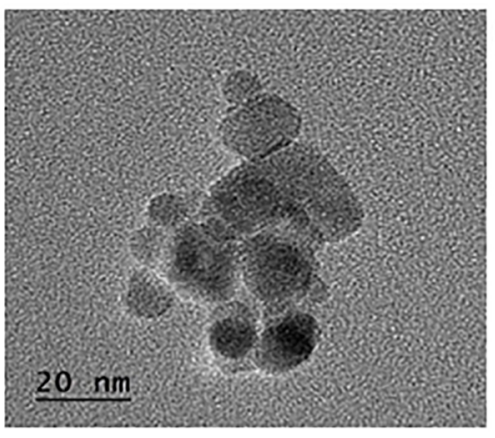

(d)

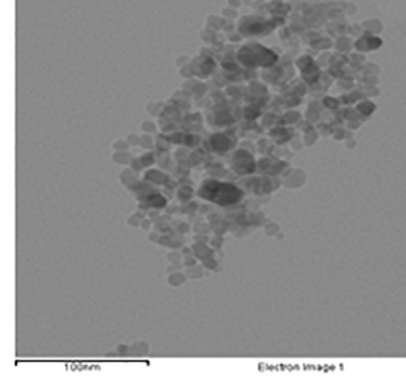

Fe Ka1

(e)

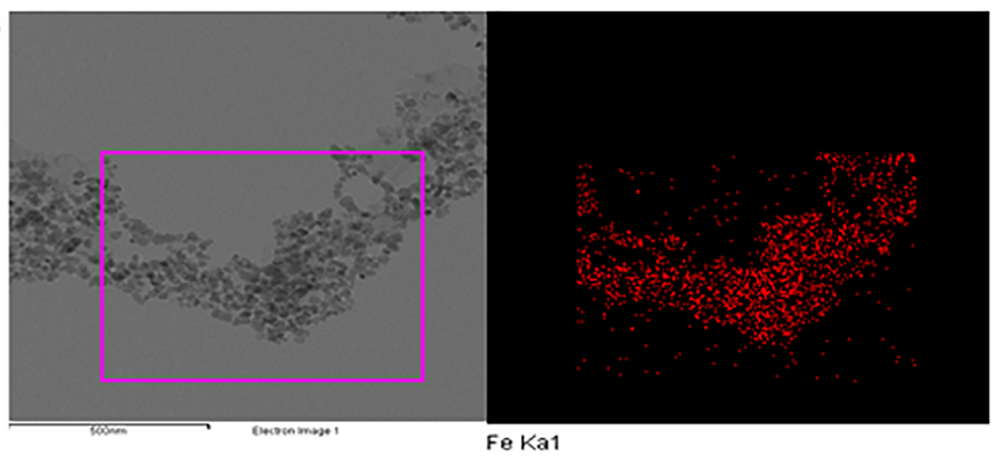

(f)

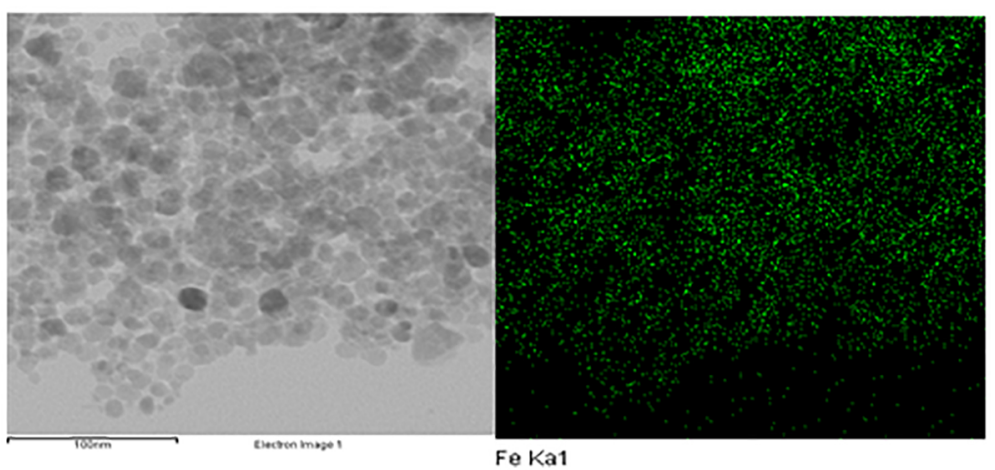

Figure 4 
(a)

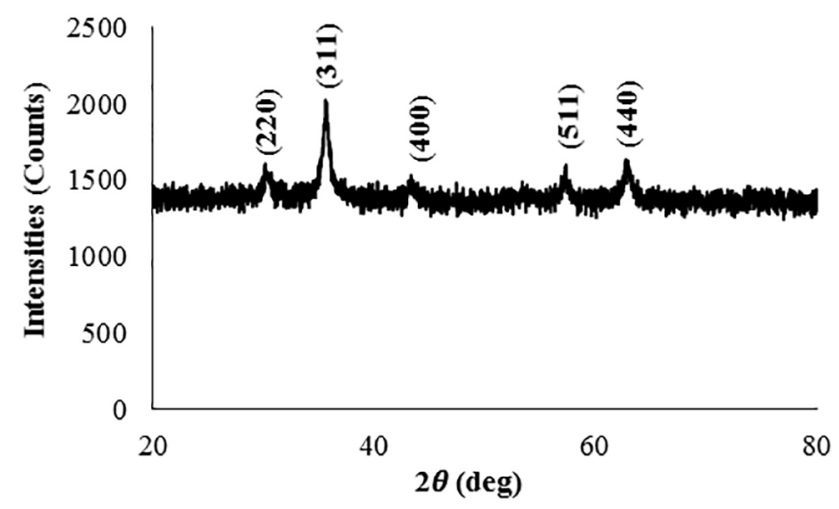

(c)

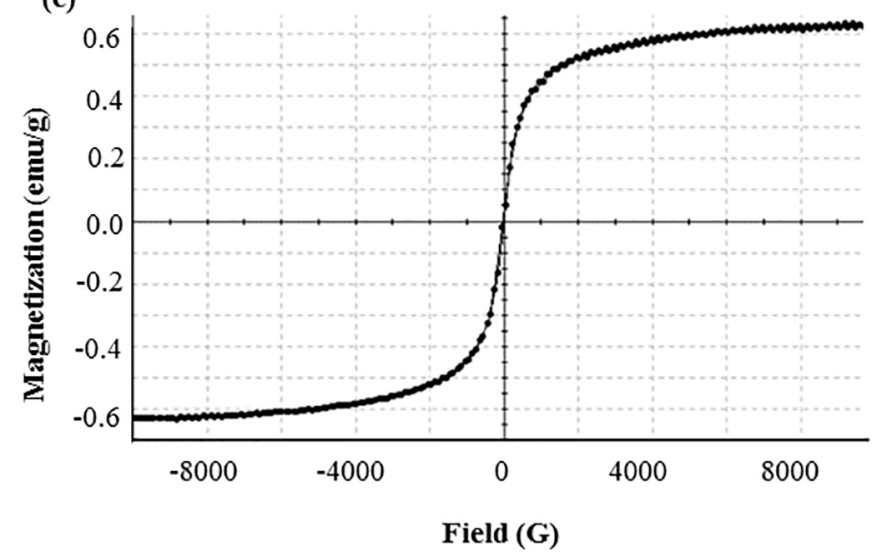

(b)
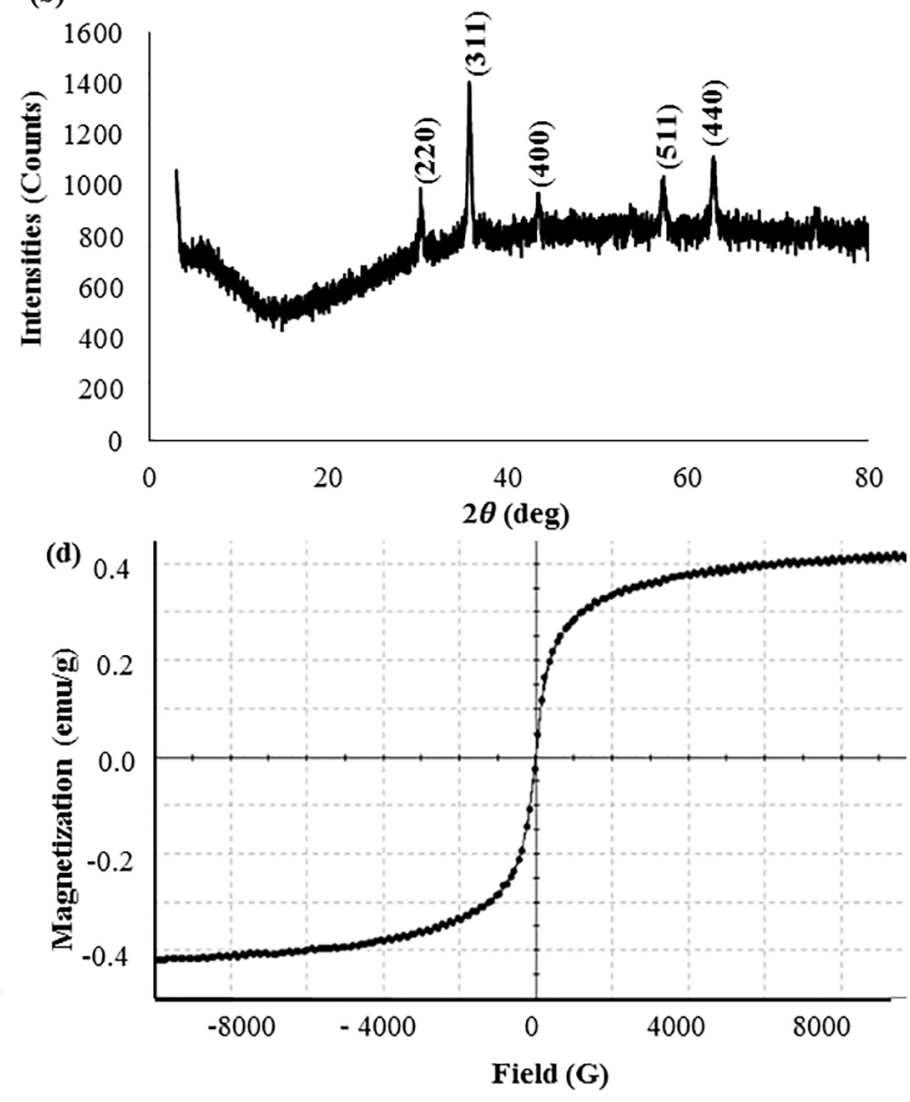

Figure 5 
(a)
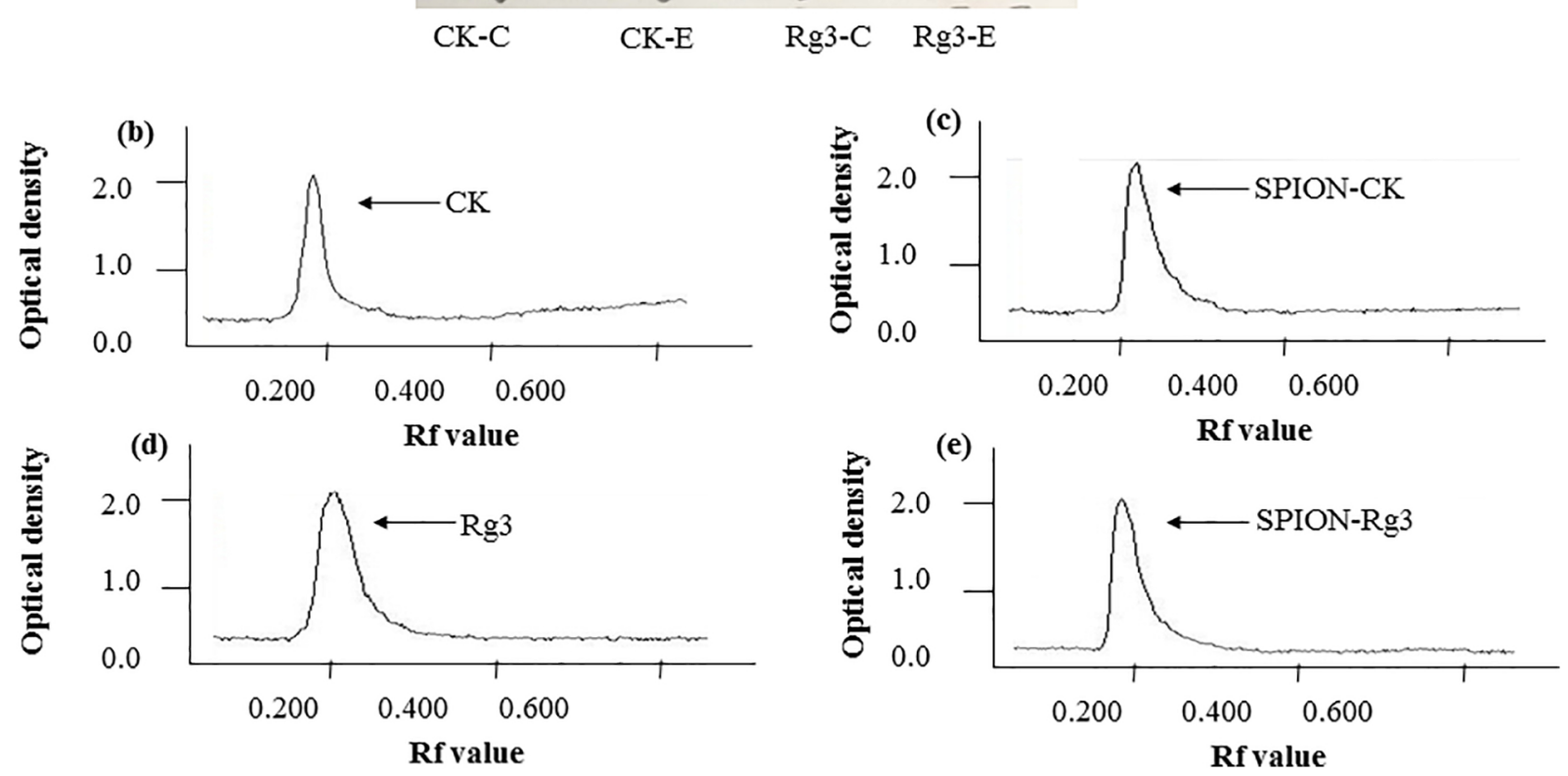

Figure 6 
(a)

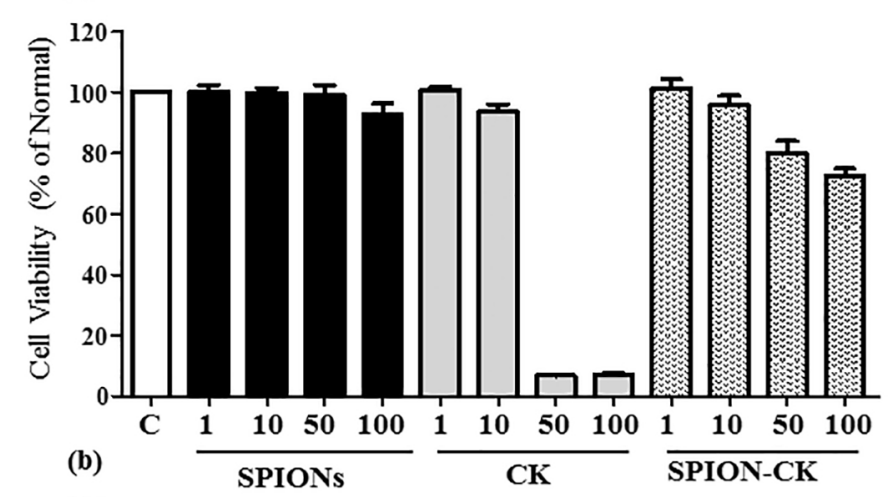

氞

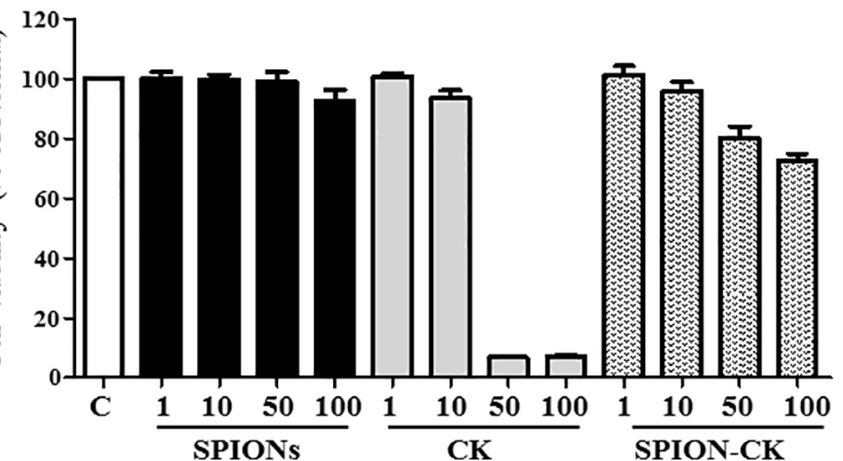

(c)
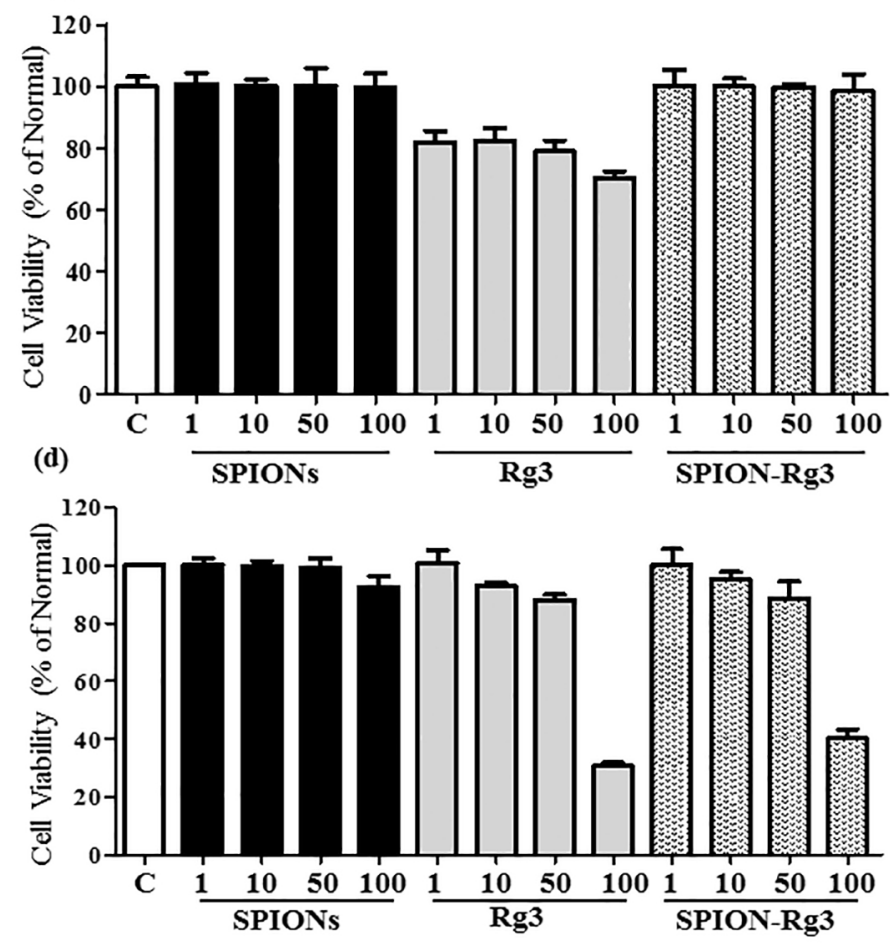


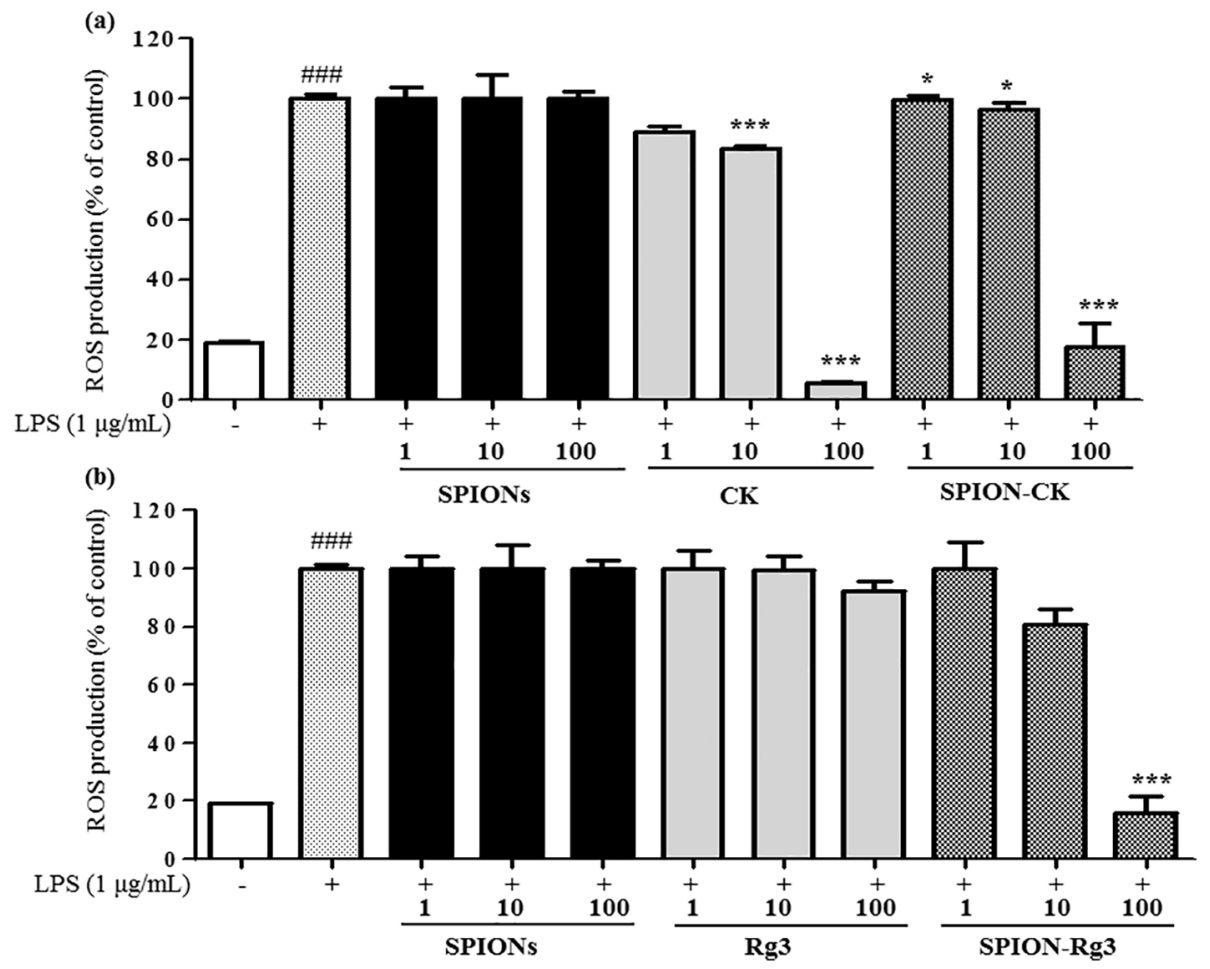

Figure 8 
(a)

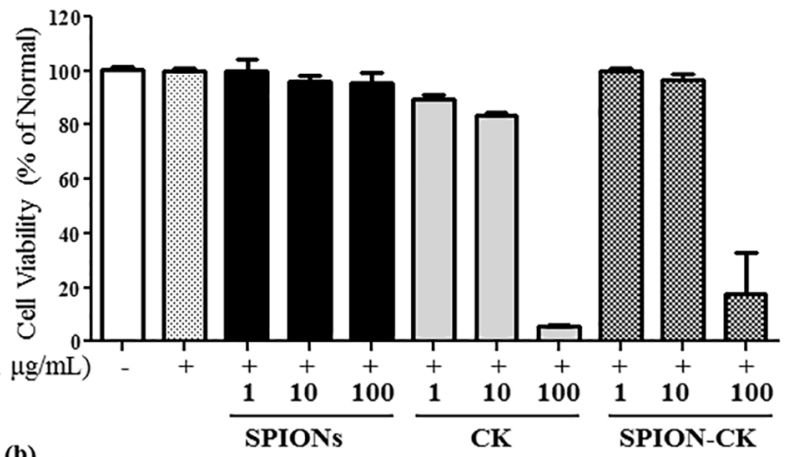

(b)

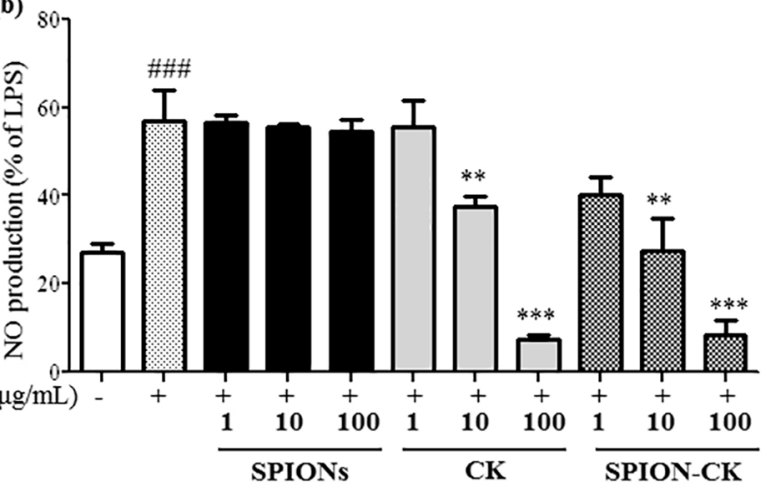

(c)

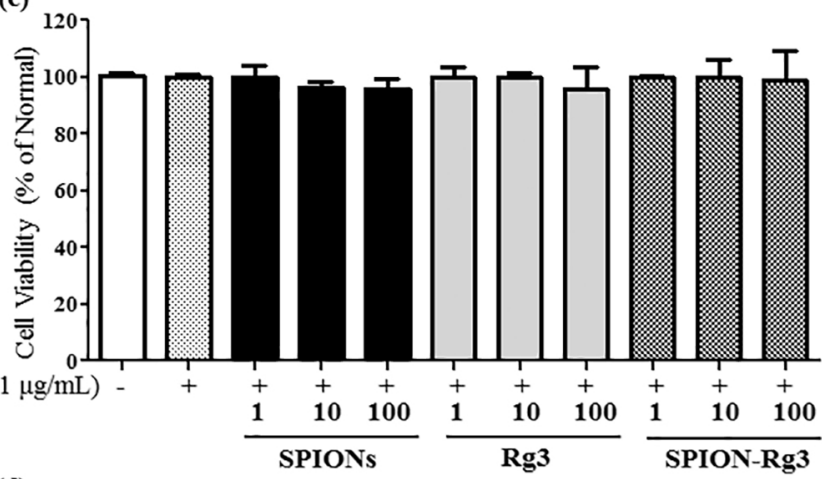

(d)

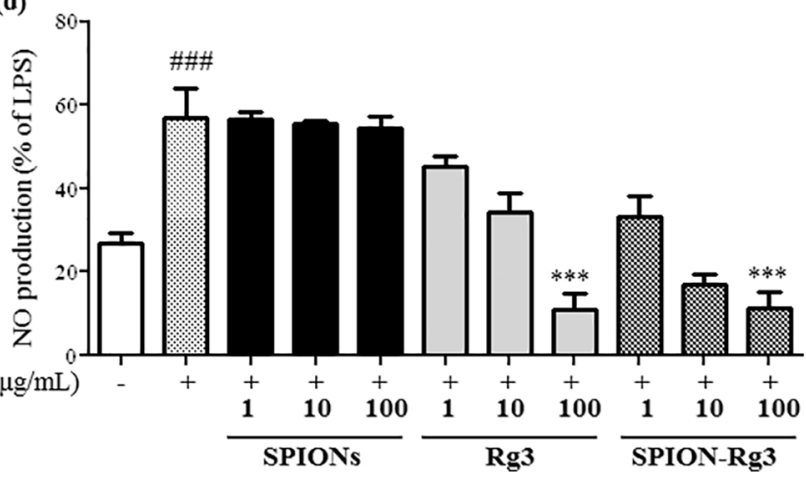

Figure 9 

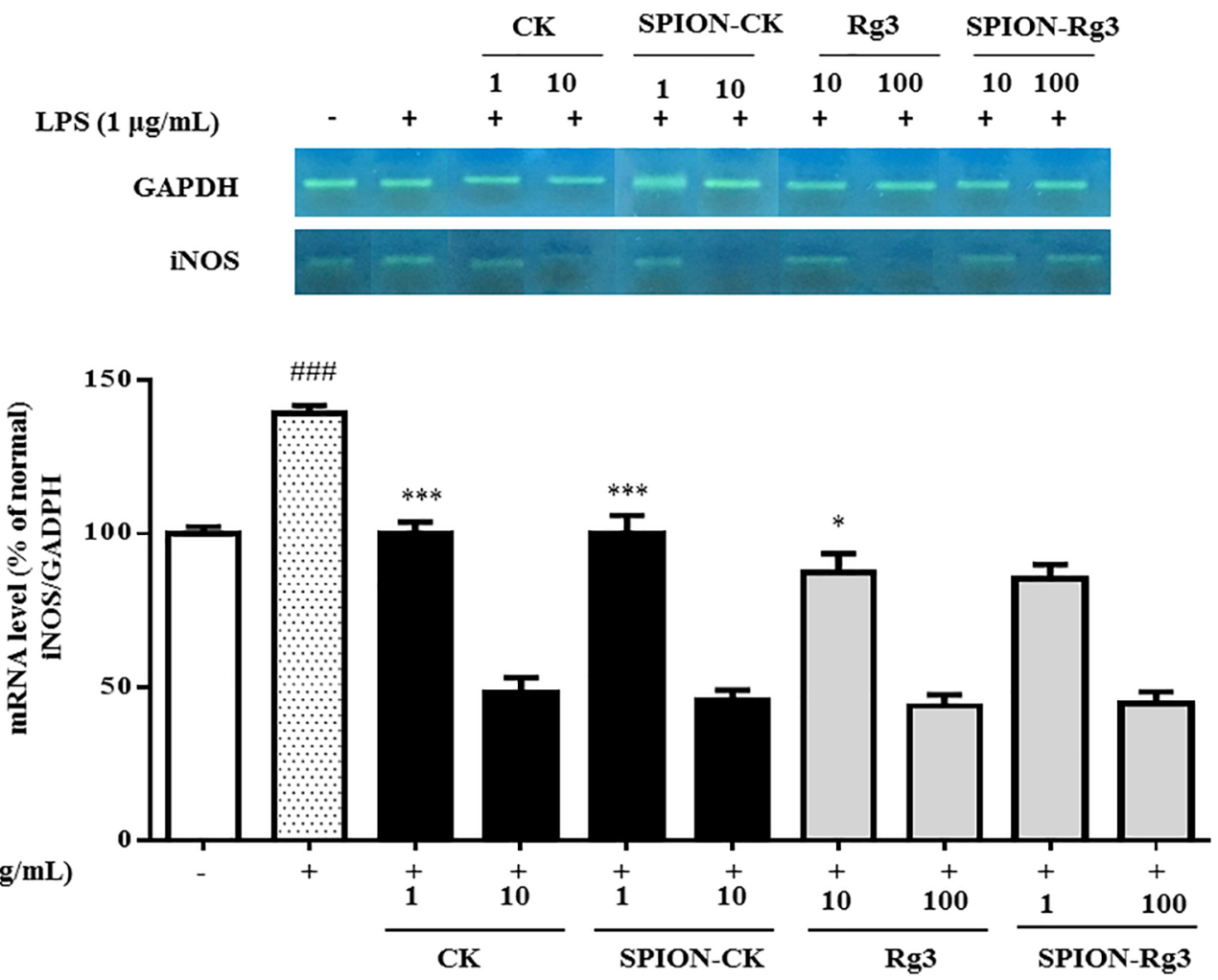

Figure 10 